\title{
Computational and Experimental Approaches for Evaluating Dose under a Block in TBI Geometry
}

\author{
Laura Russell, Jussi Sillanpaa \\ Department of Medical Physics, Memorial Sloan-Kettering Cancer Center, New York, USA \\ Email: sillanpj@mskcc.org
}

How to cite this paper: Russell, L. and Sillanpaa, J. (2022) Computational and Experimental Approaches for Evaluating Dose under a Block in TBI Geometry. International Journal of Medical Physics, Clinical Engineering and Radiation Oncology, 11, 77-83.

https://doi.org/10.4236/ijmpcero.2022.111007

Received: November 21, 2021

Accepted: February 25, 2022

Published: February 28, 2022

Copyright (c) 2022 by author(s) and Scientific Research Publishing Inc. This work is licensed under the Creative Commons Attribution International License (CC BY 4.0).

http://creativecommons.org/licenses/by/4.0/

(c) (i) Open Access

\begin{abstract}
Total Body Irradiation (TBI) patients are often treated at extended distances of several meters, with blocking made from high- $\mathrm{Z}$ materials placed close to the patients' skin. Evaluating the dose under a block (e.g., for implanted medical device shielding purposes) in such a geometry is challenging. We compare the performance of two commonly used dose calculation algorithms, Anisotropic Analytical Algorithm (AAA) and Acuros XB, with Optically Stimulated Luminescence (OSLD) and ion chamber measurements in phantoms. The calculations and phantom measurements are also compared with in-vivo OSLD measurements. We find that OSLD and ion chamber measurements in phantom are good predictors of in-vivo measurements, while both AAA and Acuros XB systematically overestimate the block transmission. We found Acuros XB to be accurate enough for a rough upper estimate (dose under block overestimated by $7 \%-22 \%)$, while for AAA the overestimate was more severe (90\%-110\%); the reason is that AAA does not account for the increase in pair production cross-section in high- $\mathrm{Z}$ materials.
\end{abstract}

\section{Keywords}

Total Body Irradiation, AAA, Acuros XB, ICD, Dose under Block

\section{Introduction}

Total Body Irradiation (TBI) patients are often treated at extended distances, with blocking made from high-Z materials placed close to the patients' skin [1] [2]. At our institution, Anterioposterior/Posterioanterior (AP/PA) beams at 440 $\mathrm{cm} \mathrm{SAD} \mathrm{are} \mathrm{employed} \mathrm{[3],} \mathrm{with} \mathrm{lung} \mathrm{blocks} \mathrm{in} \mathrm{place} \mathrm{for} \mathrm{both} \mathrm{beams.} \mathrm{Under} \mathrm{such}$ circumstances, estimating the dose under a block can be challenging. There are 
two main reasons for this. Firstly, because of the large treatment distance and nonstandard blocking and immobilization, a Computed Tomography (CT) scan in the treatment is not available (treatment planning is typically based on hand calculations). Secondly, even though the inhomogeneity corrections employed in dose calculation algorithms have become more sophisticated, modelling the absorption and scattering of Megavoltage (MV) photons in high Z-materials, such as lead or Cerrobend, remains problematic. In most Treatment Planning Systems (TPS), including Eclipse (Varian Medical System, Palo Alto, CA), the blocks, multi-leaf collimators and other beam-shaping devices in the accelerator head are modelled differently from high-Z objects in the CT image, by using a transmission factor [4]. A block placed in the accelerator head is thus modelled differently from one included in the treatment planning CT. While attenuation in the former is fairly well modelled, that in the latter often is not.

The proportion of radiotherapy patients with implanted medical devices, such as Implanted Cardiac Devices (ICDs), is increasing. ICDs are radiation-sensitive [5] and often necessitate custom radiation shielding. We investigated the suitability of different dose calculation algorithms and Optically Stimulated Luminescence (OSLD) and ion chamber measurements in designing custom shielding blocks for TBI patients with ICDs. The results of calculations and measurements performed in phantoms were compared with subsequent in-vivo OSLD measurements performed according to our standard ICD protocol.

\section{Methods}

The TBI geometry used at our institution is depicted in Figure 1. A patient with an ICD implanted in the abdomen required TBI treatment. Due to the ICD, $6 \mathrm{MV}$ was chosen for as the treatment energy, instead of the usual $15 \mathrm{MV}$. The patient was to be treated using our regular TBI geometry (AP/PA, $440 \mathrm{~cm} \mathrm{SAD,} 40 \times 40$ FS, gantry 275 , collimator 45 , patient rotates 180 degrees for AP/PA treatment), with the ICD shielded by a Cerrobend block held in a tray approx. $5 \mathrm{~cm}$ in front of the patient. Treatment Planning System (TPS) calculations, ion chamber and

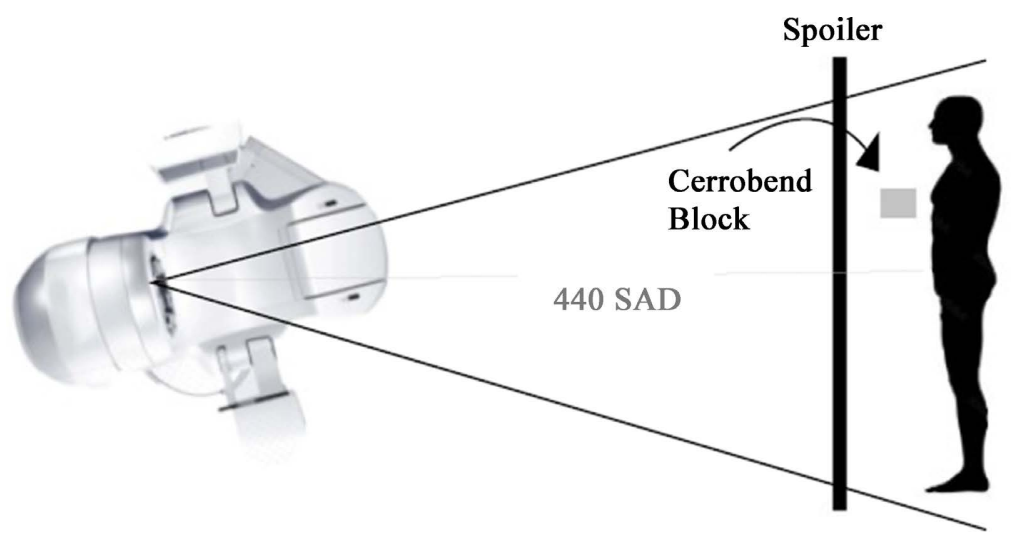

Figure 1. The TBI geometry used at our institution (the AP beam is shown; for the PA beam, the gantry angle is the same but the patient faces the other way. A separate block can be used for AP and PA if necessary). 
OSLD measurements were performed to estimate the transmission of different block designs; the results were compared with subsequent in-vivo OSLD measurements performed according to our standard ICD protocol. Patient separation at the ICD level and the AP and PA depths of the ICD $(16 \mathrm{~cm} / 2.5 \mathrm{~cm} / 13.5 \mathrm{~cm})$ were estimated from a prior CT scan. Four $7.5 \mathrm{~cm}$ thick Cerrobend blocks were evaluated; an $8 * 8 \mathrm{~cm}$ rectangular block and cylindrical blocks with 8,10 and $12 \mathrm{~cm}$ diameter.

Dose calculations were performed in Eclipse using the Analytical Anisotropic Algorithm (AAA) version 15511 and Acuros XB algorithm version 15511. A $25 \times$ $25 \times 16 \mathrm{~cm}$ virtual water phantom was created, with a virtual block $5 \mathrm{~cm}$ above the phantom. For the AAA calculations, the Relative Electron Density (RED) of the block was set to 6.97, a value generally used for Cerrobend RED [6] [7]. For Acuros XB, the high attenuation of the block meant that we had to choose the material in the Acuros XB library that was the best match for Cerrobend (stainless steel, mass density $8.00 \mathrm{~g} / \mathrm{cm}^{3}$, somewhat below the true mass density of our Cerrobend, $9.39 \mathrm{~g} / \mathrm{cm}^{3}$ ). The Acuros XB dose reporting options were set to transport in medium, dose to medium. Plans at $440 \mathrm{SAD}$ were generated for the four block designs.

Ion chamber measurements for dose under the four blocks were performed with an A12 chamber in a $25 \times 25 \times 16 \mathrm{~cm}$ solid water phantom, at the same depths as the Eclipse dose calculations (see Figure 2). Finally, OSLD measurements

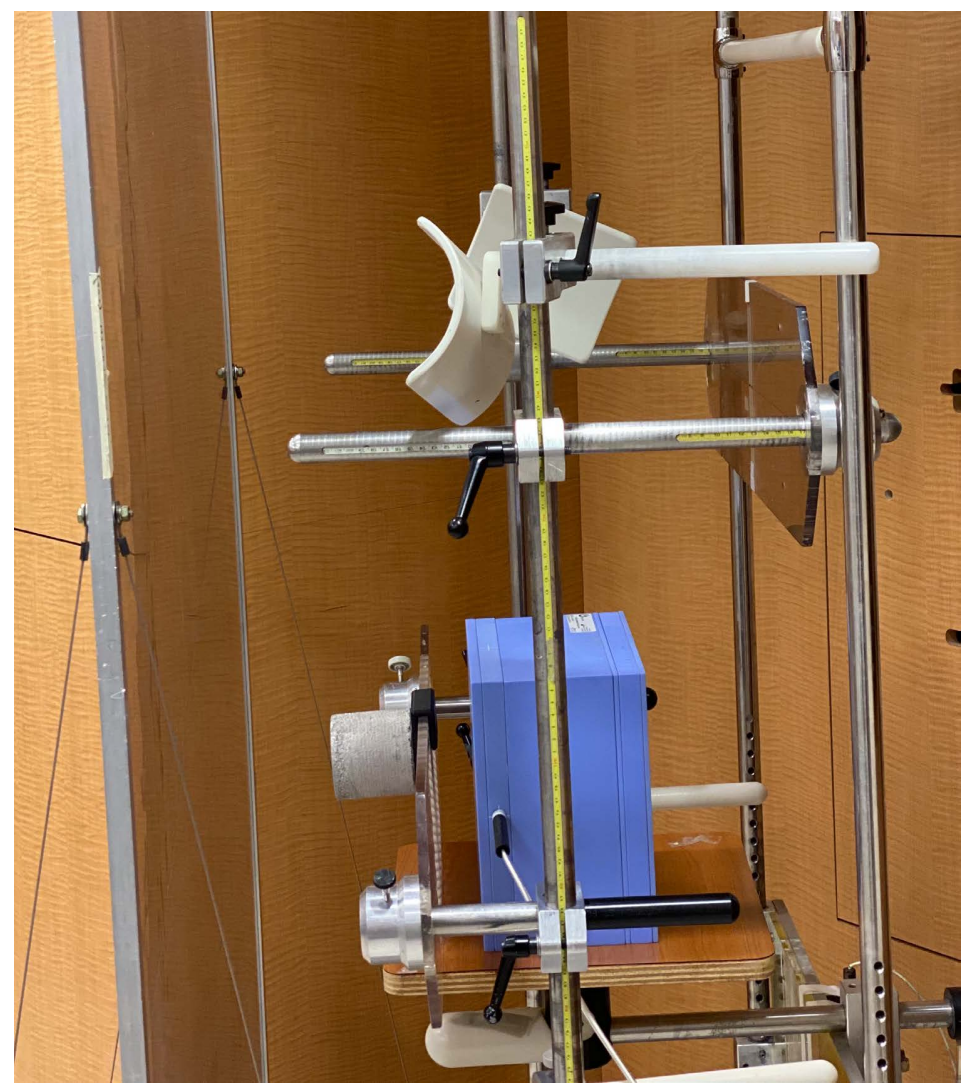

Figure 2. The ion chamber setup used for phantom measurements. 
were performed, using nanoDots (Landauer, Glenwood, Il) and the same geometry we use for in-vivo OSLD measurements on ICDs (OSLD placed on the surface of the phantom, under $5 \mathrm{~mm}$ bolus).

After a review of the results of the calculations and phantom measurements, the $8 \mathrm{~cm}$ circular block was chosen for use in the treatment for both beams as the best compromise between shielding the ICD and not reducing the dose to the parts of the patient's anatomy requiring treatment. During the first fraction, an OSLD was placed on the skin of the patient on top of the palpated ICD position, under $5 \mathrm{~mm}$ bolus.

\section{Results}

Table 1 shows the transmission for AP and PA beams in a solid water phantom for the different blocks. The calculated transmission is systematically higher than measured (both Acuros XB and especially AAA are underestimating absorption in the block). The PA transmission is larger, due to increased scatter from open parts of the field. The PA transmission is also more dependent on the size of the block (the scatter dose depends on the block size, while the direct transmission does not). Both AAA and Acuros XB model scatter in water well, therefore the agreement between measurement and calculations is better for the PA than the AP beam (especially for AAA).

Table 2 shows the measured and calculated doses in solid water at the ICD depth (on the anterior surface, under $0.5 \mathrm{~cm}$ bolus for the OSLDs) for the monitor unit (2150 MU/beam) and number of fractions (6) used in the clinical plan. The ion chamber measurements are used as the ground truth, to which AAA, Acuros

Table 1. The transmission [\%] in phantom for different block shapes for AP/PA beams.

\begin{tabular}{ccccccc}
\hline \multirow{2}{*}{ Block } & \multicolumn{2}{c}{ Ion chamber } & \multicolumn{2}{c}{ AAA } & \multicolumn{2}{c}{ Acuros } \\
\cline { 2 - 7 } & AP & PA & AP & PA & AP & PA \\
\hline 8 sq & 12.41 & 20.94 & 31.8 & 33.1 & 16.56 & 22.98 \\
8 cyl & 12.94 & 23.19 & 32.6 & 34 & 15.92 & 24.22 \\
10 cyl & 11.94 & 19.82 & 30.8 & 31.7 & 16.14 & 21.74 \\
12 cyl & 11.25 & 17.58 & 29.5 & 29.9 & 16.56 & 19.57 \\
\hline
\end{tabular}

Table 2. The total predicted ICD dose in solid water for the clinical MU/beam and number of fractions. The difference between the ion chamber measurements and other methods is also presented.

\begin{tabular}{cccccccc}
\hline Block & $\begin{array}{c}\text { Ion chamber } \\
\text { (cGy) }\end{array}$ & $\begin{array}{c}\text { AAA } \\
\text { (cGy) }\end{array}$ & \% Diff & $\begin{array}{c}\text { Acuros } \\
\text { (cGy) }\end{array}$ & \% Diff & $\begin{array}{c}\text { OSLD } \\
\text { (cGy) }\end{array}$ & \% Diff \\
\hline $8 \mathrm{sq}$ & 190.4 & 379.8 & 99.5 & 217.9 & 14.4 & 197.2 & 3.6 \\
$8 \mathrm{cyl}$ & 205.1 & 389.9 & 90.1 & 219.3 & 6.9 & 211.3 & 3.0 \\
$10 \mathrm{cyl}$ & 181.6 & 365.5 & 101.3 & 209.3 & 15.2 & 185.5 & 2.2 \\
$12 \mathrm{cyl}$ & 165.8 & 348.3 & 110.1 & 202.1 & 21.9 & 167.4 & 1.0 \\
\hline
\end{tabular}


Table 3. The in-vivo OSLD dose reading (scaled for the 6 FX treatment course), compared with the dose predictions in phantom.

\begin{tabular}{cccccc}
\hline Block & $\begin{array}{c}\text { Ion chamber } \\
\text { (cGy) }\end{array}$ & $\begin{array}{c}\text { AAA } \\
(\mathrm{cGy})\end{array}$ & $\begin{array}{c}\text { Acuros } \\
(\mathrm{cGy})\end{array}$ & $\begin{array}{c}\text { Patient OSLD } \\
(\mathrm{cGy})\end{array}$ & $\begin{array}{c}\text { OSLD } \\
(\mathrm{cGy})\end{array}$ \\
\hline $8 \mathrm{cyl}$ & 205 & 390 & 220 & 204 & 211 \\
\hline
\end{tabular}

$\mathrm{XB}$ and OSLDs are compared. The ion chamber dose is reduced, by approx. $24 \%$, as the block diameter is increased from $8 \mathrm{~cm}$ to $12 \mathrm{~cm}$. This is due to reduced scatter under the block, especially in the PA beam. The AAA results are systematically too high by $90 \%-110 \%$. The Acuros XB results are also systematically too high, but by a smaller factor $(7 \%-22 \%)$. The OSLD results closely track the ion chamber results and are only $1 \%-4 \%$ higher.

Table 3 compares the OSLD measurement during patient treatment to pretreatment calculations and measurements in solid water, for the whole 6 fraction treatment course. There is good agreement between the patient treatment OSLD measurement and both the OSLD and ion chamber measurements in solid water phantom. The Acuros XB calculation is $8 \%$ higher than the patient OSLD, while AAA overestimates the dose by $91 \%$.

\section{Discussion}

Both OSLD and, especially, the ion chamber measurements in solid water agreed well with the in-vivo OSLD measurements. Both methods are useful for estimating the OSLD dose.

TPS calculations are less accurate than measurements, but faster and less labor intensive to perform. Both Acuros XB and AAA systematically underestimated the attenuation in Cerrobend blocks, but Acuros XB performed significantly better than AAA. The reason for this is that while AAA scales the dose deposition and scatter kernels calculated in water by the relative electron density [8] [9], Acuros $\mathrm{XB}$ utilizes a library of interaction cross-sections accounting for Compton scatter, photoelectric effect and pair productions for different materials [10] [11]. If the mass density is below $3 \mathrm{~g} / \mathrm{cm}^{3}$, a mixture of the cross-sections of the two closest materials in the library is automatically applied and the mass density may be specified by the user; if it is above this threshold $\left(9.38 \mathrm{~g} / \mathrm{cm}^{3}\right.$ for Cerrobend), the user has to select a material from the library, with no adjustment of the mass density (the closest choice is stainless steel, $8.0 \mathrm{~g} / \mathrm{cm}^{3}$ ). AAA is therefore accounting accurately for changes in the Compton scattering cross-section, which is proportional to electron density, but not for the changes in the photoelectric (cross-section per atom proportional to $\mathrm{Z}^{4}$ ) or pair production (cross-section per atom proportional to $Z^{2}$ ) cross-sections [12]. AAA performs well in water-like materials where the Compton scattering dominates, but significantly underestimates the attenuation in Cerrobend since the increase in pair production is not properly accounted for.

The accuracy of the Acuros XB calculations could be improved further, if the 
mass density of the block could be set to the true value of $9.38 \mathrm{~g} / \mathrm{cm}^{3}$ while keeping the stainless steel interaction cross sections or, preferably, Cerrobend was added to the Acuros XB material library. We have submitted a product improvement request to Varian Medical Systems.

We found that Acuros XB calculations were accurate enough to give a clinically useful rough estimate of the dose to the ICD; the results were approx. $7 \%-22 \%$ higher than the ion chamber measurements. This is a significantly better agreement than in the previous work by Lamichane and Studenski [7]. They compared AAA and Acuros XB calculations with ion chamber measurements in a similar geometry (400 SSD, $2 \mathrm{~cm}$ thick, $10 \times 10 \mathrm{~cm}$ Cerrobend block $2 \mathrm{~cm}$ in front of a $30 \times 30 \times 30 \mathrm{~cm}$ solid water phantom, filed size $10 \times 10 \mathrm{~cm}$ ). Their conclusion was that for $5 \mathrm{~cm}$ depth, the dose calculated by Acuros XB was 37\% too high and that by AAA $52 \%$ too high. Their general conclusion (TPS calculations underestimate the attenuation in the block, more so for AAA than Acuros XB) is the same as in our work and the AAA results match ours closely. The reason for the relatively poor performance of Acuros XB in their work is not known, but may be related to the method of material and mass density assignment.

Our result indicate that ion chamber measurements in a solid water phantom are a good method of predicting dose to an ICD in the TBI geometry, and Acuros $\mathrm{XB}$ calculations could be used as a very rough upper estimate. Acuros XB could also be used as a rough guide in estimating the effect of blocks at extended distances in other similar situations (e.g., immobilization devices with metal parts, spleen or lung blocks in TBI treatments).

\section{Conclusion}

The ion chamber and OSLD measurements in solid water predicted the results of the patient measurements well. Acuros $\mathrm{XB}$ was accurate enough to provide a rough estimate, while AAA overestimated the dose to ICD significantly. The Acuros $\mathrm{XB}$ results could be improved further by adding more high- $\mathrm{Z}$ materials to the cross-section library in the algorithm.

\section{Funding}

This research was supported in part by NIH Core Grant P30 CA008748.

\section{Conflicts of Interest}

The authors declare no conflicts of interest regarding the publication of this paper.

\section{References}

[1] Van Dyk, J., Galvin, J.M., Glasgow, G.P., et al. (1986) The Physical Aspects of Total and Half Body Photon Irradiation. AAPM Report No. 17, American Association of Physicists in Medicine.

[2] Wong, J., Filippi, A.R., Dabaja, S., Yahalom, J. and Specht, L. (2018) Total Body Irra- 
diation: Guidelines from the International Lymphoma Radiation Oncology Group (ILROG). International Journal of Radiation Oncology · Biology · Physics, 101, 521-529. https://doi.org/10.1016/j.ijrobp.2018.04.071

[3] Shank, B. (1983) Techniques of Magna-Field Irradiation. International Journal of Radiation Oncology · Biology · Physics, 9, 1925-1931.

https://doi.org/10.1016/0360-3016(83)90364-4

[4] Varian Medical Systems (2021) Eclipse Photon and Electron Algorithms Reference Guide. Varian Medical Systems, Palo Alto, CA, USA. https://www.myvarian.com/

[5] Miften, M., Mihailidis, D., Kry, S.F., et al. (2019) Management of Radiotherapy Patients with Implanted Cardiac Pacemakers and Defibrillators: A Report of the AAPM TG203. Medical Physics, 46, e757-e788. https://doi.org/10.1002/mp.13838

[6] Spirydovich, S., Papiez, L., Langer, M., Sandison, G. and Thai, V. (2006) High Density Dental Materials and Radiotherapy Planning: Comparison of the Dose Predictions Using Superposition Algorithm and Fluence Map Monte Carlo Method with Radiochromic Film Measurements. Radiotherapy \& Oncology, 81, 309-314.

https://doi.org/10.1016/j.radonc.2006.10.010

[7] Lamichhane, N. and Studenski, T. (2020) Improving TBI Lung Dose Calculations: Can the Treatment Planning System Help? Medical Dosimetry, 45, 168-171. https://doi.org/10.1016/j.meddos.2019.09.004

[8] Ulmer, W., Pyyry, J. and Kaissl, W. (2005) A 3D Photon Superposition/Convolution Algorithm and Its Foundation on Results of Monte Carlo Calculations. Physics in Medicine \& Biology, 50, 1767-1790. https://doi.org/10.1088/0031-9155/50/8/010

[9] Tiilikainen, L., Helminen, H., Torsti, T., et al. (2008) A 3D Pencil-Beam-Based Superposition Algorithm for Photon Dose Calculation in Heterogeneous Media. Physics in Medicine \& Biology, 53, 3821-3839. https://doi.org/10.1088/0031-9155/53/14/008

[10] Vasilliev, O.N., Wareing, J., Damis, A.M., et al. (2008) Feasibility of a Multigroup Deterministic Solution Method for Three-Dimensional Radiotherapy Dose Calculations. International Journal of Radiation Oncology · Biology · Physics, 72, 220-227. https://doi.org/10.1016/j.ijrobp.2008.04.057

[11] Han, T., Mikell, J.K., Salehpour, M. and Mourtada, F. (2011) Dosimetric Comparison of Acuros XB Deterministic Radiation Transport Method with Monte Carlo and Model-Based Convolutional Methods in Heterogeneous Media. Medical Physics, 38, 26512664. https://doi.org/10.1118/1.3582690

[12] Johns, H.E. and Cunningham, J.R. (1983) The Physics of Radiology. 4 Edition, Charles C Thomas, Springfield. 